\title{
MEDICAL DATA COMPRESSION AND TRANSMISSION IN NOISY WLANS: A REVIEW
}

\author{
Mustafa Almahdi Algaet ${ }^{1 *}$, Abd Samad Bin Hasan Basari ${ }^{2}$, Ali Ahmad Milad ${ }^{3}$, \\ Salem Msaoud Adrugi ${ }^{4}$ and Salem Mustafa Aldeep ${ }^{5}$ \\ 1,3,4,5 Department of Computer, Faculty of Education, Elmergib University, \\ Alkhums, Libya \\ ${ }^{2}$ Department of System and Computer Communication, Faculty of Information \\ and Communication Technology Universiti Teknikal Malaysia Melaka Durian \\ Tunggal Malaysia \\ 1'malgaet@elmergib.edu.ly, ${ }^{2}$ abdsamad@utem.edu.my, ${ }^{3}$ ali106014@gmail.com, \\ ${ }^{4}$ salem.adrugi@elmergib.edu.ly and ${ }^{5}$ sm771984@gmail.com
}

\begin{abstract}
Data compression and transmission are becoming increasingly important in the field of computer communication. These techniques are commonly used in medical communities for data exchange in hospital computer networks. Computer-based modeling applications serve as possible approaches in designing easy-to-evaluate medical data communications. This study aims to develop a simulation tool that manages medical data compression and transceiver over noisy Wi-Fi IEEE 802.11b. This paper briefly introduces communication networks and services, wireless communication concepts, transmission technologies, network topologies in hospitals, medical information technologies, and wireless telemedicine systems. This paper also reviews related research, especially studies on data compression algorithms and data transmission standards on Wi-Fi with past, present, and future directions.
\end{abstract}

Keywords - WI-FI, IEEE 802.11b, Medical Data, Compression and Transceiver, ICA, DCT, SPIHT and JPEG2000

\section{INTRODUCTION}

Telemedicine describes medical services support that uses telecommunications. The prefix "tele" refers to distance and originated from ancient Greek language. Hence, telemedicine literally means providing medical services over a certain distance. In medical applications, usage of telecommunications includes delivery of medical information between pairs of transmitters and receive [1].Medical information can be as simple as a doctor providing consultation to refined data obtained from a human body.

Telemedicine ensures delivery of required medical advice over long distances, especially in rural areas, through the usage of telecommunication, which is economical. Telemedicine applications utilize multimedia equipment and telecommunication systems to deliver medical care, diagnosis, consultation, and treatment along with transmission of education and medical information. Telemedicine normally involves physicians who use interactive videos and/or store-and-forward consultations during treatment of patients.

Interactive videos enable medical specialists to directly communicate with remote patients through monitors and specifically modified gadgets. Store-and-forward methods comprise physicians who send pictures, X-rays, and patient information straight to a

Received: May 24, 2019

Reviewed: December 9, 2019

Accepted: December 12, 2019

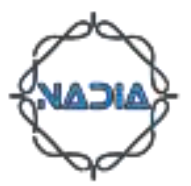


specialist's computer. After analyzing received information, the specialist subsequently sends diagnosis back to the local doctor who treats patients and ensures availability of follow-up care[2].

In recent years, portable and mobile personal computers and workstations followed an increasing trend. Constantly increasing numbers of mobile users demand access to network services comparable with their "tethered" equivalents. Wireless systems and networks enable communication between mobile terminals and allow access to highcapacity wired networks through these mobile devices. In satisfying wireless data networking requirements, formation of study group 802.11 was initiated under the Institute of Electrical and Electronics Engineers (IEEE) project 802-recommended wireless local area network (WLAN) international standards. The 802.11 study group aimed to develop media access control (MAC) and physical layer (PHY) in wireless connectivity with fixed, portable, and mobile station standards within local areas. PHYs that must be considered include direct-sequence spread spectrum (DSSS), frequencyhopping spread spectrum (FHSS), and diffuse infrared.

\subsection{COMMUNICATION NETWORKS AND SERVICES}

Communication networks support various healthcare and medical services. Telemedicine utilizes numerous network categories to allow physicians to share ideas; surgeons in any part of the world can simultaneously perform operations regardless of operating locations; nurses and paramedics can retrieve a patient's record regardless of time and location. Hospitals and clinics utilize networks in taking care of patients to manage work and inventory. This section discusses basics of telecommunication technology, with emphasis on wireless networking, because telemedicine applications need the flexibility provided by wireless networks.

\subsubsection{WIRELESS COMMUNICATIONS BASICS}

Telecommunication refers to the exchange of information among various units. Communication systems comprise transmitters (sender), receivers (recipient), and channels (the path where information travels). Information is transmitted in a timely manner via the communication channel, where the recipient receives information at a time. This procedure appears simple when sent and received information feature similarities[3] and [4]. However, such case is not true always. Unfortunately, channels are degraded, for example, by additive noise, distortion, and attenuation [1]. Figure 1 shows that additive noise is introduced to and becomes part of information. In certain ways, the addition of noise corrupts the original information. In telemedicine systems, transfer of information over a certain distance can be limited to several micrometers inside devices or integrated circuit chips or thousands of kilometers across continents.

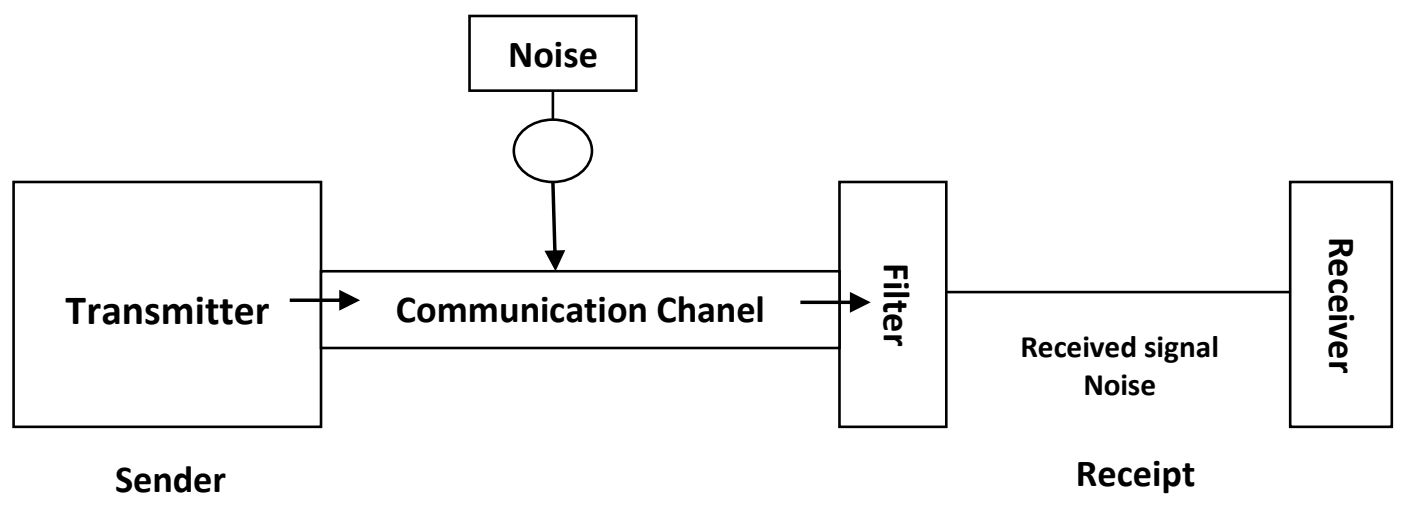

Fig. 1 Communication system under noise presence 


\subsubsection{WIRED VERSUS WIRELESS}

Wireless communication systems became popular because of improvements in technology, which efficiently resolves many dependability and security issues that normally restrict usage of wireless technology in cost-saving applications. Agility and accessibility are dynamic factors in selecting wireless form of communication. Although wired and wireless communications are used widespread, these types of communications feature specific differences.

Wired communications were used for more than a century. Resulting from the invention of telegraphy in mid-19th century, telephony innovations started when Bell and Gray developed the first telephone by utilizing a microphone to pick up a person's voice, which is reproduced by a speaker. This invention shaped the base of electric wires utilized in telecommunications. Prior to this event, wired communication started in early 1794 when C. Chappe delivered visual telegraphs through a line-of-sight communication channel.

Wireless technology began in 1887 , the same time as introduction of the first telephone. This technology was developed when Hughes and Hertz generated radio waves with a park gap transmitter. At the end of 19th century, fundamental wireless technology molded radio broadcasting base by Faraday and Marconiat. Three decades after introduction of the first radio, television broadcasting emerged in 1930s and was followed by introduction of commercial-licensed television stations in Pennsylvania and New York in 1941. In 1984, the first electromechanical television was invented in Germany. Thus far, radio and television broadcast are equally one-way communication systems and are identified as simplex communications. During World War I, utilization of two-way radio communication was evident, but its commercial usage started after World War II. Although the US patent for wireless telephone belonged to Stubblefield in 1908, cellular phones became widely accessible in 1980s when the Federal Communications Commission authorized the Advanced Mobile Phone Service system[5]. Up to now, the major progression of wireless communications may be unclear to end users as it only allows them to communicate with one another without any additional structures.

The 2G Global System for Mobile communications (GSM) was launched in Europe in 1991 and supported text messaging since 1993. Afterward, 2.5G and 3G emerged with a selection of fresh structures, for example video call, Multimedia Messaging Service, and Internet surfing. Speed of wireless technologies advancement over the past decades can be possibly visualized. Subsequently, evolution of wired and wireless technologies spans more than a century and is now considered as matured technologies [1].

Interestingly, both wired and wireless technology is also categorized as 'guided' and 'unguided' media, respectively, respectively. Figure 2 illustrated the flowchart of guided versus unguided transmission medium. Information running along a cable is 'guided' through a fixed path which is the cable itself. On the other hand, in wireless communication, information does not under a fixed guidance, hence it is known as 'unguided'. In conclusion, based on its usage in telemedicine application, for short distance transmission, wired communication is more reliable and cheaper. Wireless communication is more convenient as it has a high mobility and flexibility in transmission. Telemedicine applications normally use wireless technology as mobility is highly sought after- everyone despises the idea of having wires tangling all over the body. 

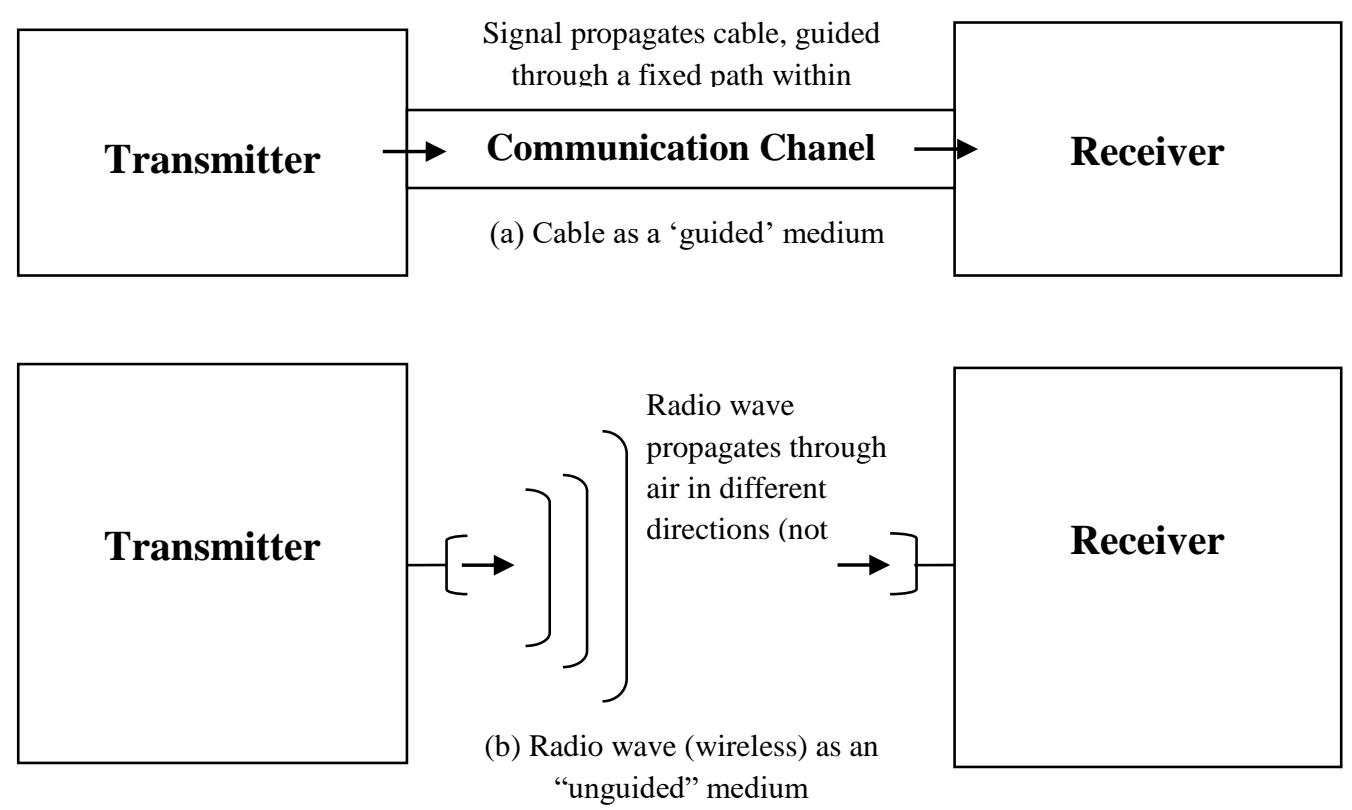

Fig. 2 Guided versus unguided transmission medium

\subsubsection{DATA TRANSMISSION SPEED}

The amount of information transferred in channels is determined by bandwidth, which serves as an important terminology in understanding topics related to communications. As channel bandwidth is fixed, the general rule is that high data rates are supported by equivalently high data rate.

With fixed transmission medium bandwidth, increasing rate of transmitted data becomes possible by adding numerous bits into one baud. Baud is defined as a count of the number of changes in electronic states per second. As an illustration, $1 \mathrm{~K}$ baud copper cable alters the voltage 1000 times per second. However, this cable does not essentially allow transmission of 1000 data bit per second. Bandwidth describes transmitted data rate supported by specific channels. The reference occupies frequency bands of electronic signals during data transmission across channels. Consequently, channel bandwidths are measured in hertz $(\mathrm{Hz})$, and variance between maximum and minimum frequencies are often used. For instance, transmission of voice data through telephone channels measures between 300 (minimum frequency) and $3400 \mathrm{~Hz}$ (maximum frequency) with a bandwidth of $3.1 \mathrm{KHz}$.

\subsubsection{ELECTROMAGNETIC INTERFERENCE (EMI)}

The main deficiency of wireless communications involves EMI; negative effects of EMI cause more problems compared with conducting cables. This deficiency is mainly a risk in healthcare applications as wireless transmitting devices can affect operations of fragile medical instruments. Explained various methods to reduce effects of EMI on healthcare applications to protect operational reliability. Among available solutions, proper shielding was provided by using suitable housing material for medical instruments; it can efficiently protect devices from unnecessary interferences as the susceptibility of all wireless transmitting devices to EMI from adjacent radiating sources cannot be treated not seriously. 


\subsubsection{MODULATION}

Modulation is a process known as "carrier signal"; it provides the required energy for sending information to receivers. This process changes in certain ways based on information that it carries. Modulation also creates input of data and transforms them into signals that are ready for transmission. Altering specific carrier signal parameters changes representative data. For instance, in frequency modulation, radio broadcasting frequencies of carrier signals are modified when they concern voice information. Variance in frequency is interpreted by the receiver (radio) as the voice is carried over. In complicated modulation schemes, transformation or changes may occur in more than one parameter simultaneously to ensure that considerable information is represented per baud, thus increasing transmission efficiency [6].

\section{TRANSMISSION TECHNOLOGY}

\subsection{DATA TRANSMISSION}

Data transmission refers to the physical transfer of data through communication channels, for example, wires, wireless technologies, or physical media. Transmission area can cover either interior or exterior of hospitals or large areas, for example, between countries. Hospitals utilize transmission of medical data to support transfers among other facilities, which may possibly or may not be on the same network.

In addition to direct contact with patients, data transmission can immediately deliver medical findings to physicians in communities. Doctors use this technology to obtain instant access to data, as opposed to waiting for the arrival of physical media. Doctors also gain access to a patient's medical history, thus improving point of care service. Patients can electronically receive their data exams, and they do not need to carry and store physical media. Physicians can access data in various locations and have their data ready in advance.

With the publication of European Telecommunications Standards Institute as the first WLAN standard, Hyper-LAN/1 was confirmed in 1995, whereas Hyper-LAN/2 was established in 2000. By contrast, the standard of IEEE 802.11 WLAN became widely accepted. Movable devices, such as laptops, personal digital assistants, and mobile phones, feature standardized and built-in 802.11 chipsets. Wireless infrastructure equipment (access points (APs)) is moderately low-priced.

WLAN technology rapidly progresses. The original IEEE 802.11 standard sustains data rates up to $2 \mathrm{Mb} / \mathrm{s}$. At present, devices commonly feature $54 \mathrm{Mb} / \mathrm{s}$ capabilities. Devices utilizing multiple inputs, multiple outputs (MIMO) technology, which can support up to $300 \mathrm{Mb} / \mathrm{s}$, are becoming more popular. The success of 802.11 standards is attributed to integrating improvements in modulation methods while preserving interoperability with legacy schemes. However, new modulation schemes do not control successive schemes [7]. 802.11 can select any scheme from updated modulation schemes to optimize frame transmission. In this manner, wireless devices show potential to link rates and to adapt to channel conditions.

\subsection{TYPE OF WIRELESS NETWORKS}

Development of wireless communications, such as Bluetooth, IR, Wi-Fi, ZigBee, cellular networks, WiMAX (fixed), and Local Multipoint Distribution Service, reached an extent where several options are available. These various network types enhanced many applications with range of coverage spanning several meters to thousands of kilometers. Table I specifies and summarizes several frequently used network properties in telemedicine applications. 
Table I. Properties of Some Common Wireless Systems

\begin{tabular}{|l|c|c|c|}
\hline Network Type & Frequency Range & Speed & Maximum Range \\
\hline Bluetooth & $2.4-2.485 \mathrm{GHz}$ & $3 \mathrm{Mbps}$ & $300 \mathrm{~m}$ \\
\hline IR & $100-200 \mathrm{THz}$ & $16 \mathrm{Mbps}$ & $5 \mathrm{~m}$ \\
\hline Wi-Fi & $2.4-5 \mathrm{GHz}$ & $108 \mathrm{Mbps}$ & $100 \mathrm{~m}$ \\
\hline ZigBee & $900 \mathrm{MHz}$ & $256 \mathrm{Kbps}$ & $10 \mathrm{~m}$ \\
\hline Cellular Networks & $850-1900 \mathrm{MHz}$ & $20 \mathrm{Mbps}$ & $5 \mathrm{~km}$ \\
\hline WiMAX (Fixed) & $10-66 \mathrm{GHz}$ & $1 \mathrm{Gbps}$ & $10 \mathrm{~km}$ \\
\hline LMDS & $10-40 \mathrm{GHz}$ & $512 \mathrm{Mbps}$ & $5 \mathrm{~km}$ \\
\hline
\end{tabular}

Wide use of IEEE 802.11 or Wi-Fi standards in wireless home networks is inexpensive and suitable for Internet access. When compared with Bluetooth and IR, WLANs require more development to set up initial configurations prior to establishing communication links. Common IEEE 802.11 standards comprise $\mathrm{a} / \mathrm{b} / \mathrm{g} / \mathrm{n}$; definitions of these standards account for PHY specifications (PHY defining transmission of raw data bits over air) and MAC layer of WLANs (provides and addresses channel access control procedures to allow several devices to connect with a single AP).

A simple WLAN consists of no less than one AP and mobile client(s) (MCs). MCs are primarily any mobile device that attempts to find and preserve wireless connection to networks via APs. APs are placed in various locations throughout the coverage area to form wireless network infrastructure. Installation of many APs increases network coverage area. Installing a wireless relay can aid extending range of coverage. Existence of multiple APs within vicinities allows MCs to select APs with strong signal strength.

Derived from IEEE 802.11 WLAN by the Wireless Ethernet Compatibility Alliance, Wi-Fi provides a unified standard for diverse types of wireless devices. Wi-Fi is occasionally referred to as Wireless Internet. Wireless devices serve as APs or as "hotspot". Wi-Fi technology is regularly utilized in off-site monitoring of patients recovering at home as current home networks can be employed with minimal changes [8].

\subsection{OVERVIEW OF IEEE 802.11 WLAN}

\section{IEEE 802.11}

IEEE 802.11 or Wi-Fi signifies a set of WLAN standards advanced by functioning for the IEEE LAN/metropolitan area network (MAN) Standards Committee's group 11 of the IEEE LAN/MAN Standards Committee. IEEE 802.11 WLAN standard approval and compatible standard specifications were distributed in 1997. After legacy standard was finalized, numerous standard amendments were released, and other current revisions were discussed in working groups of IEEE 802.11. The standard composition comprises PHY and MAC specifications for WLANs. New PHY specifications allow the use of high data rates (e.g., up to $11 \mathrm{Mbps}$ in 802.11b and $54 \mathrm{Mbps}$ in 802.11a as compared with 1 and 2 Mbps in the initial version, as summarized in Table II.

Table II. IEEE 802.11 Standards Overview [9]

\begin{tabular}{|c|c|c|}
\hline Standards & Description & Ratification \\
\hline 802.11 & $\begin{array}{l}\text { Based definition of MAC; IR, FHSS, and DSSS in } 2.4 \mathrm{GHz} \\
\text { band PHYs with } 1 \text { and } 2 \text { Mbps data rates }\end{array}$ & $\begin{array}{l}\text { 1997; amended 1999, } \\
2007\end{array}$ \\
\hline $802.11 \mathrm{a}$ & $\begin{array}{l}\text { OFDM PHY in } 5.8 \mathrm{GHz} \text { UNIII spectrum, data rates up to } 54 \\
\text { Mbps }\end{array}$ & 1999 \\
\hline $802.11 b$ & $\begin{array}{l}\text { DSSS PHY extension in } 2.4 \mathrm{GHz} \text { band for data rates up to } 11 \\
\text { Mbps }\end{array}$ & 1999 \\
\hline $802.11 n$ & $\begin{array}{l}\text { Wireless network bearer operating in the } 2.4 \text { and } 5 \mathrm{GHz} \text { ISM } \\
\text { bands with data rates up to } 600 \mathrm{Mbps}\end{array}$ & 2009 \\
\hline $802.11 \mathrm{ac}$ & $\begin{array}{l}\text { Wireless network bearer operating below } 6 \mathrm{GHz} \text { to provide } \\
\text { data rates of at least } 1 \mathrm{Gbps} \text { per second for multi-station } \\
\text { operation and } 500 \mathrm{Mbps} \text { on a single link }\end{array}$ & $\begin{array}{l}\text { Targeted for } 2012 \text { at } \\
\text { present }\end{array}$ \\
\hline
\end{tabular}




\section{802.11 PHYS}

The 802.11 PHYS are separated into two sub-layers, namely, Physical Layer Convergence Procedure (PLCP) and Physical Medium Dependent (PMD) sub-layers. The PLCP sub-layer encapsulates MAC protocol data units (MPDUs) from the MAC layer into frames in transmission by a PMD unit. The PMD sub-layer implements transmission encoding schemes. MPDUs are registered to PLCP service data units (PSDUs), which in turn, are compressed into PLCP PDUs. PMD identifies a number of PHYs to support multiple modulation schemes. PPDU formats differ based on PHY. The innovative 802.11 standard identifies three PHYs: FHSS, DSSS, and IR.

Back transmission speeds of FHSS and DSSS reach 1 and $2 \mathrm{Mb} / \mathrm{s}$ on IR PHY. Introduction of high-rate DSSS in the $802.11 \mathrm{~b}$ revision supports speeds of up to $11 \mathrm{Mb} / \mathrm{s}$. As opposed to spreading spectrum, 802.11a and 802.11g use orthogonal frequencydivision multiplexing (OFDM), which provides speeds of up to $54 \mathrm{Mb} / \mathrm{s}$. 802.11n integrates battered OFDM approaches with MIMO technology. 802.11n can attain transmission rates of $600 \mathrm{Mb} / \mathrm{s}[10]$.

\section{IEEE 802.11b}

Application of first-generation DSSS or FHSS of IEEE 802.11 standard provided 1 Mbps differential binary phase shift keying (DBPSK) modulation or 2 Mbps differential quadrature phase-shift keying (DQPSK) modulations. IEEE $802.11 \mathrm{~b}$ became accessible in 1999. This design was carried out in indoor environments and offered a maximum of $11 \mathrm{Mbps}$ in a common unlicensed industrial, scientific, and medical $2.4 \mathrm{GHz}$ radio band.

Barker sequence utilization codes the sequence for 1 and $2 \mathrm{Mbit} / \mathrm{s}$. long direct sequence specification describes two supplementary data rates (5.5 and $11 \mathrm{Mbit} / \mathrm{s})$. Hence, products and chipsets were developed without difficulty to support 802.11b enhancements. The substantial increase in throughput in $802.11 \mathrm{~b}$ combined with substantially reduced price led to the rapid acceptance of $802.11 \mathrm{~b}$ as the final WLAN technology. $802.11 \mathrm{~b}$ is generally used in the point-to-multipoint configuration, in which communication of AP with one or many clients through an omnidirectional antenna occurs in coverage area around it. The protocol is also utilized in externally securing point-to-point measures with high-gain antennas. The range normally reaches up to $8 \mathrm{~km}$ regardless of favorable reports within ranges of $80-120 \mathrm{~km}$, where the establishment of a line of sight is possible. The IEEE $802.11 \mathrm{~b}$ divides the spectrum into 14 overlapping and staggering channels with $5 \mathrm{MHz}$ center frequencies.

No specification for channel width is provided for standards of $802.11 \mathrm{~b}$. Relatively, specifications are presented for center frequency and spectral mask of channels. The $802.11 \mathrm{~b}$ spectral mask needs weak signals at least $30 \mathrm{~dB}$ from the peak energy at \pm 11 $\mathrm{MHz}$ and from center frequencies, those that decrease by no less than $50 \mathrm{~dB}$ from the aforementioned peak energy at $\pm 22 \mathrm{MHz}$. As spectral mask describes limitations of power output, which reaches $\pm 22 \mathrm{MHz}$ from the center frequency, the general assumption is that energy of channels fails to extend further than these parameters. When the transmitter is adequately influential, signal strength can reach beyond the $\pm 22 \mathrm{MHz}$ point. Consequently, based on separation of channels 1,6 , and 11, any channel signals can be adequately weakened to minimize interference with a transmitter on any other channel. Hence, channels 1, 6, and 11 are realized as non-overlapping. When transmitters are collectively nearer than channels 1,6 , and 11 (for example 1, 4, 7, and 10), overlapping among channels possibly causes unacceptable dilapidation of signal quality and throughput [11]. 


\section{IEEE 802.11a}

The standard 802.11a employs parallel core procedure as a novel standard operation in $5 \mathrm{GHz}$ band, and usage of 52 subcarriers OFDM with concentrated $54 \mathrm{Mbit} / \mathrm{s}$ raw data rate yields realistic net, which is an attainable throughput in mid-20 Mbit/s. Data rate reduction starts from 48, 36, 24, 18, 12, 9, and $6 \mathrm{Mbit} / \mathrm{s}$ when necessary. 802.11a possesses 12 non-intersecting channels, with eight intended for the interior and four assigned to point-to-point connections. Out of 52 subcarriers, four are selected as pilot tones to correct residual frequency offset errors that accumulate over symbols. PHY inserts physical preamble of the data frame and moderate's data frames that are coded at a MAC-quantified data rate. Usage of physical preamble allows receivers to perceive the beginning of packet transmission and to coordinate with the transmitter's clock. IEEE 802.11 MAC avoids collisions as a result of concurrent Carrier Sense Multiple Access/Collision Avoidance transmissions. A total of 52 subcarriers are used by systems, which are moderated via BPSK, QPSK, 16QAM or 64QAM. Forward error correction coding (convolutional coding) is used at coding rates of $1 / 2,2 / 3$, or $3 / 4$ [12].

\section{HOSPITAL NETWORK TOPOLOGY}

At the end of $20^{\text {th }}$ century, computers and technology field generally accepted open concepts. Thus, new interoperable platforms were developed, and various healthcarefocused technologies were adopted, for example, roentgenography computerization, computed tomography, sonography scanning, and X-ray communications tools. Similarly, centralized computer systems are reduced, and tools, such as electronic medical charts, began operating on standard software, such as Windows and UNIX. Currently, the above-mentioned healthcare apparatuses can connect to single network systems, which can exchange data in any type of platform. Figure 3 shows a sample of a hospital network connected to all medical equipment inside a hospital. Hospital networks are also connected to computers, which are operated by the administrator, nurses, doctors, and other healthcare staff [13]. Several requirements are needed to determine a healthcare network: network bandwidth and quality of service, redundancy and preventive measures against system failures; security, efficiency, and flexibility of network infrastructure.

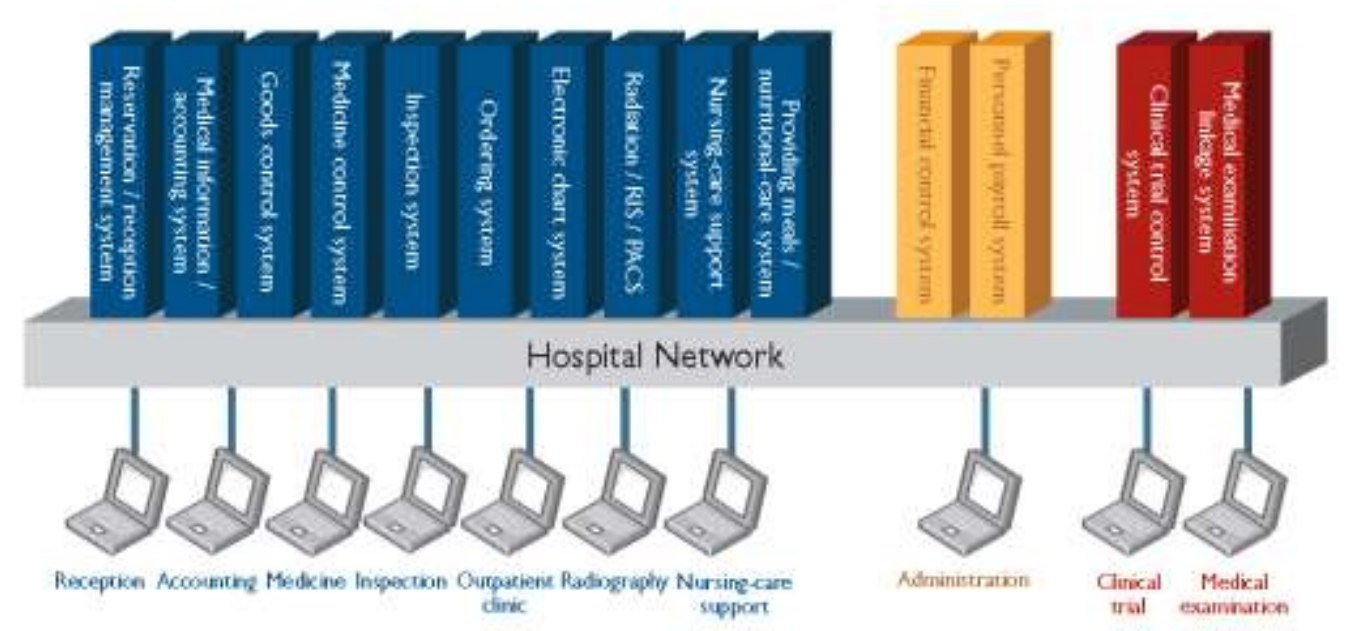

Fig. 3 Hospital network connecting all infrastructure including medical apparatus, administration and clinical doctors [13]

\subsection{WLAN IN HOSPITAL}

A hospital usually comprises patient rooms. Usage of WLAN technology increases as the public utilize laptops and other devices. As a hospital amenity, WLAN service is a 
current approach for providing Internet access to inpatients. Sufficient security actions must be considered when implementing an open LAN environment[14]. Figure 4 illustrates an example of implementation of WLAN in a hospital network.

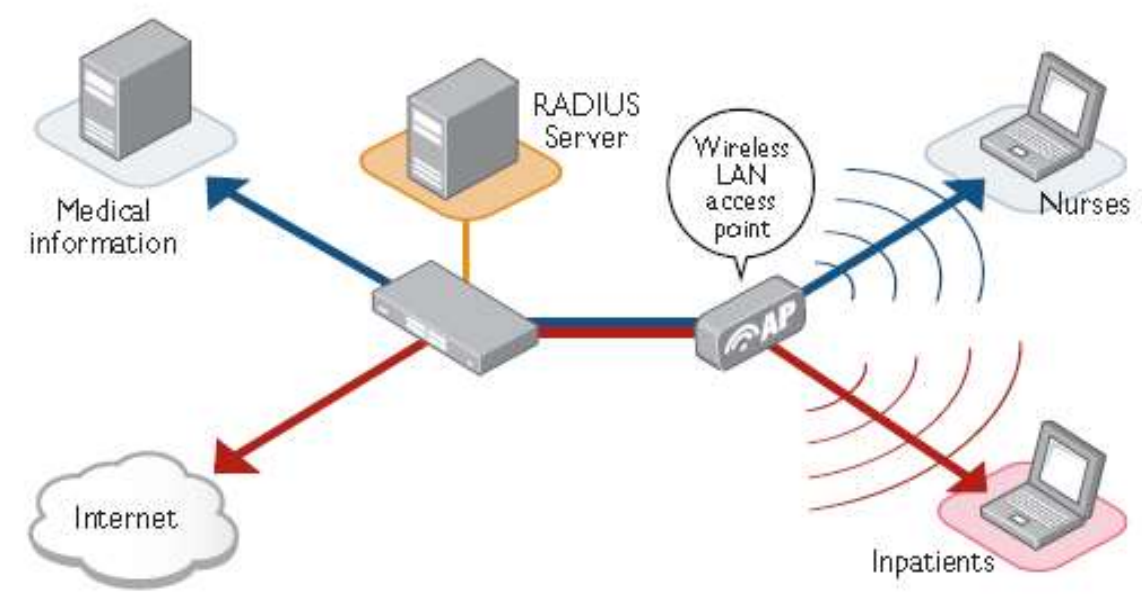

Fig. 4 Example of WLAN implementing in hospital network that facilitating either healthcare staff or inpatients [15]

\subsection{HOSPITAL NETWORKING}

With the current wireless technology, healthcare professionals can access patient records regardless of locations via connection to information systems of hospitals. However, several restrictions on present technologies generally depend on General Packet Radio Service (GPRS) technologies and utilization strategies in health care. These issues are as follows:

1. Absence of obtainable flexible link in various mobile communication choices and health care facility criteria.

2. Expensive communication links.

3. Absence of current e-Health services and additional information systems, for example, integrated medical records.

Recently, the emergence of wireless technologies, namely, WLAN and Zigbee was reported in mobile health services. WLAN reaches high speeds of up to $54 \mathrm{Mb} / \mathrm{s}$ in data networks, which are accessible to consumers provided that they are within covered range (characteristically 30-50 m indoors and 100-500 m outdoors) of a WLAN base station. Bandwidths with high data rates are delivered by WLAN to provide voice and multimedia communications without any risk of signal interference with medical equipment. WLAN permits mobile communications within a medical site and enables medical staff to access medical information systems regardless of differing locations.

Zigbee standard is a new short-range, low-power, low-rate wireless network (IEEE 802.15.4 based) that complements high-data-rate technologies, for example, WLAN and various new applications. Zigbee standards can be utilized in various applications in medical hospital settings, for example, patient body area network and information exchange among medical devices.

In conjunction with wireless technologies, such as WLAN and Zigbee, portable handheld devices permit staff to rapidly access applications, for example, instant messaging, mobile multimedia conferencing, and doctor/patient localization. Challenges of hospital sensor networking include the following: 
1. As a result of monitoring patients through wireless networks, engagement with sensor nodes in hospitals will increase significantly in the future. Integration of these nodes in huge numbers should easily form an ad-hoc networks that provide flexibility.

2. Meeting actual restrictions particularly throughout emergency situations.

3. Long battery life for sensor nodes to provide comfort to patients.

4. Creating energy-saving sensor nodes.

5. Addressing safety qualities such as patient's identity.

Hospital area network (HANs) are designed primarily with WLAN and Zigbee. HANs feature heterogeneous network design because they consist of low-power Zigbee network for monitoring health sensor nodes, medical instruments, PCs at nurse stations, and convenient handheld gadgets. Interconnection of ZigBee medical network to other medical services occurs via WLAN APs. WLAN allows usage of many complex communication protocols, such as Transmission Control Protocol/Internet Protocol, for Intranet/Internet connections inside hospitals or other co-operational medical facility centers. Development of a Zigbee-ready mote includes a processor-radio board comprising Texas Instruments MSP430 microcontroller linked to Chipcon CC2420 2.4 $\mathrm{GHz}$ transceiver over Synchronous Peripheral Interface. The transceiver is connected to 2.4 GHz GigaAnt Rufa left antennas via balun circuits. The proposed Zigbee ready mote is used as the hardware platform for medical sensor nodes and devices because of the following:

1. Possibility of attaining one-year battery life.

2. Small size that is comfortable for use.

3. Zigbee provides a security toolbox, which is founded on Advanced Encryption Standard to ensure reliable and secure networks.

\section{TECHNOLOGIES IN MEDICAL INFORMATICS}

\subsection{MEDICAL DATA FORMAT}

The format of standardized medical data indicates organizing and storing such digital information. In general, images, signals, and videos are significant information frequently used in medical applications. The primary format for image data is DICOM, which is standard for handling, storing, printing, and transmitting information in medical imaging. This type of information includes a file format description and network communication protocol. DICOM is an acronym for Digital Imaging and Communications in Medicine.

DICOM files can be exchanged between two entities capable of receiving image and patient data in DICOM format. DICOM allows integration of scanners, servers, workstations, printers, and network hardware from multiple manufacturers into a picture archiving and communication system. DICOM represents a kind of metadata-data format that contains a structure obtaining image information, other patient information, and medical identification.

Formats of the medical data signal or medical time-series recordings include BioSemi data Format (BDF) and European Data Format (EDF). These formats can simultaneously store multiple signal channels, time-stamped annotations, and meta-information. These formats are applied in electroencephalography, polysomnography, electromyography, evoked potentials, electro-neurography, and electrocardiography. BDF and EDF are also types of metadata - data formats that contain a structure acquiring signal information, other patient information, and medical identification [16]. 


\subsection{MEDICAL IMAGING}

Medical imaging is a method used in creating human body images for clinical uses (parts and organ functions). Medical imaging methods produce large amounts of data, specifically from CT, magnetic resonance imaging (MRI), and positron emission tomography modalities. Therefore, storing electronic image data and communications necessitate compression. JPEG2000 is the new version of compressing DICOM-standard images to store and transmit medical images. Price and possibility of accessing huge image data sets over low or numerous bandwidths are discussed further by using another DICOM standard, known as JPEG 2000 Interactive Protocol, which enables effective streaming of JPEG 2000 compressed image data [17].

\subsection{IMAGE COMPRESSION}

Image compression is used to address the issue of reducing the amount of information in representing the digital image. This procedure is proposed in yielding the compact representation of an image, thus reducing the required image storage space in transmission. Each image contains redundant data. Redundant data refer to duplicated data in images; such data may be pixels repeated across images or patterns. Image compression occurs by using redundant information on images. Reducing redundancy aids in saving storage space for images. Image compression results in the reduction or elimination of one or more of these redundancies. Compression requires removing one or more of these basic data redundancies. Three basic data redundancies can be identified or utilized in image compression (Inter-pixel Redundancy, Coding Redundancy, and Psycho-Visual Redundancy) which are:

I. Interpixel Redundancy: As a result of adjacent correlation of images, adjacent image pixels are statistically not self-determining. This form of redundancy is known as interpixel redundancy. This particular redundancy is occasionally referred to as spatial redundancy, and it can be explored through several ways, one of which is to predict a pixel value based on adjacent pixel values. With such purpose, mapping novel 2-D array pixels is achieved through different formats, for example, various ranges between adjacent pixels. When reconstruction of pixels of the original image is based on altered data set, reverse mapping becomes possible.

II. Coding Redundancy: Made up of changeable length code, words are selected in matching of statistics of original sources. In such cases, the image itself or pixel value becomes the processed version. This particular coding is constantly reversible; it typically implements and utilizes lookup tables. Huffman codes and arithmetic coding technique are samples of image coding scheme that explore coding redundancy.

III. Psycho-Visual Redundancy: Various experiments on psychophysical aspects of human vision confirmed that the human eye does not react with equivalent sensitivity to all incoming visual information; several information pieces are significant. Majority of the image coding algorithms utilized today take advantage of this redundancy category, for instance, the DCT-based algorithm at the core of JPEG encoding standard.

Compression benefits:

1. Include saving costs in several data sent over switched telephone networks, where call cost is frequently based on duration.

2. Reduction involves storage requirements at entire execution time.

3. Transmission error probability is reduced as few bits are transmitted. 
4. Security level against illegal monitoring is provided based on image compression methods as two subsequent main categories: lossless image compression and lossy image compression.

Compression of an image with lossless compression involves encoding the original file information. Hence, decompressed images are precisely identical from the original. Lossless image compression samples include Portable Network Graphics and Graphics Interchange Format (GIF). Utilizing a specific image compression format depends on images compressed. Lossless image compression methods include the following schemes:

- Run-length encoding (RLE): Is a straightforward image compression type that runs stored data as single-data value and count rather than as original run. This scheme is used for sequential and recurring data. This method substitutes identical symbol (pixel) sequences known as runs, hence performing RLE lossless image compression. RLE is commonly applied in fax machines.

- Entropy encoding: Refers to a self-sufficient; lossless data compression for precise traits of a medium. One entropy-coding main type involves creating and assigning a distinctive prefix-free code for every unique symbol occurring in an input. Compression of images by entropy encoder replaces each fixed-length input symbol with equivalent variable-length prefix-free output codeword.

- Huffman Encoding: This was developed by Huffman, is an entropy-encoding algorithm utilized for lossless data compression. To date, Huffman coding is frequently utilized as a "back-end" step to several compression processes. Reference addresses usage of variable-length code tables to encode source symbols; derivation of variable-length code table is based on the expected possibility of occurrence of the possible value of source symbols. Pixels of images are treated as symbols and regularly assigned to small number of bits, whereas symbols occurring rarely are assigned to a comparatively large number of bits. A prefix code indicates that any symbol (binary) code is not the code for any other symbol.

- Arithmetic coding: An entropy encoding utilized in lossless data compression. In general, a string of characters represents the words "hello there," utilizing a fixed number of bits per character, as indicated in the American Standard Code for Information Interchange code. A string conversion to arithmetic encoding regularly uses characters stored with small bits and rarely occurring characters stored with numerous bits, resulting in usage of several bits in total. Arithmetic coding differs from other entropy encoding forms, for example, compared with Huffman coding, rather than separating inputs into component symbols and replacing them with a code, arithmetic coding encodes the whole message into a single number.

- Lempel-Ziv-Welch (LZW): Which was created by Abraham Lempel, Jacob Ziv, and Terry Welch, is a universal lossless data compression algorithm. As a dictionary-based coding, LZW is static or dynamic. The dictionary is fixed in static dictionary during encoding and decoding. This dictionary is updated during dynamic dictionary coding. Implementation of the algorithm is simple and features perspective in for highthroughput hardware implementations. LZW is a widely utilized UNIX file compression algorithm and is also applied in GIF image format. In computers, LZW compression became the first widely utilized universal image compression method. LZW was once used to compress a large English text file to about half its original size.

Lossy compression, as implied by the name, leads to some information loss. With reference to image lost, the compressed image is not the same as the original uncompressed image after several rounds of information compression. This method characteristically suits images. JPEG is a typical example of lossy compression. Any 
algorithm restoring a presentation identical to the original image is identified as lossy technique. Image reconstruction is an estimation of the original image; as a result, image quality must be measured for lossy compression techniques. Lossy compression provides high compression ratio compared with lossless compression. Performance considerations in lossy compression schemes mainly include:

1. Compression ratio

2. Noise ratio signal.

3. Encoding and decoding speed.

The following schemes are examples of lossy image compression techniques:

1. Scalar Quantization: Scalar quantization is a common form of quantization, which is characteristically identified as $\mathrm{Y}=\mathrm{Q}(\mathrm{x})$, where the procedure uses quantization function $\mathrm{Q}$ in mapping a scalar (one-dimensional) input value $\mathrm{x}$ to a scalar output value Y. Scalar quantization is basic and natural as rounding high-precision numbers to adjacent integer or to adjacent multiple of several other precision units.

2. Vector Quantization (VQ): is a conventional quantization method based on signal processing; it allows modeling of probability density functions by distributing sample vectors. Utilized initially to compress image, VQ works with division of a large set of points (vectors) into groups in order of similar number of points adjoining them. VQ density matching property influences identification of large and high-dimensioned data density. Considering that representation of data points is by their closest centroid index, low-error and rare high-error data are frequently observed. Thus, VQ is appropriate for lossy data compression because of its practicality for data correction and density estimation [18].

When an image is reduced for transmission or storage, the aim is to improve the efficiency of transmission or to save space. However, most compressed data algorithms are lossy, indicating that information on the original image is not conserved to enable recovery of the processed image by decompression; the processed image is not similar to the initial one prior to compressing. However, in a lossless compression, processed images can revert to the original form after compression without losing any details or clarity. This condition indicates that no variance should be detected when two images are compared before and after compression and decompression. A digital image comprises a group of pixels with a long sequence of " 0 's" and " 1 's". Lossless and lossy compression approaches as reviewed by tutorial. Compressing medical images improves the efficiency of transmission over telemedicine networks and reduces storage cost for mass electronic patient records.

Compressing transpires by locating areas in images where colors are similar; these areas are then marked as "similar colors are detected in this area." Compression eliminates gaps, empty fields, and redundancy within images. Compressed medical images usually contain large amounts of sensitive and significant details, which make lossy compression inappropriate. These details are areas restrained from being identical in color or shade of gray and are easily lost due to compression.

In numerous medical images, details are represented by delicate colors and grey-shade differences, which may be too refined to be discerned by the naked eye and contain important information on the state of health of patients. These details include circumstances, for example, primary data of a cancerous tumor or fetus with abnormality. Compressed lossy image algorithms may discard weak details when necessary. Quality factor describes the quality depth of image degradation. Some related works are briefly presented in the following:

An algorithm that develops speed for multispectral images was presented by. This algorithm comprises huge redundancy in search, significantly decreasing encoding speed and reducing space and time requirements. With the intention of solving speed issues, the 
authors presented a quick algorithm search that scans wavelet coefficient matrix when determining the significance of all required $\mathrm{D}(\mathrm{i}, \mathrm{j})$ and $\mathrm{L}(\mathrm{i}, \mathrm{j})$ when executing SPIHT. Block-based pass-parallel SPIHT is another algorithm that disintegrates wavelettransformed images into $4 \times 4$ blocks and concurrently encodes bits in a bit-plane of a $4 \times$ 4 block.

[19] suggested five measures in improving quality of compressed images. First, a new tree with virtual root was introduced to maintain wavelet coefficients. Second, an extra matrix was used to enhance speed and significance and the decision of trees. Third, preprocessing flattened coefficients prior to encoding SPIHT. Fourth, predictable bits were eliminated from encoder output by reorganizing the coding process. In conclusion, the balance of quantization from the middle-point is based on statistics. Experiments revealed that these developments intensify peak signal-to-noise ratio (PSNR) up to $5 \mathrm{~dB}$ at very low rates, and normal enhancement at $0.2-1 \mathrm{bpp}$, which reaches $0.5 \mathrm{~dB}$ for standard test images, is utilized. The complexity of computation is also reduced.

Another study suggested an original scheme to improve robust transmission of SPIHTbased color image coder over noisy channels. This scheme can be applied when SPIHT bit streams are reorganized based on their represented spatial domains (square block) without losing coding efficiency. Slices or group of blocks are transferred independently. Another version of SPIHT was introduced as a fast and simple hardware.

A state table with four bits per coefficient was used instead of lists to maintain a set partitions and encoded information. ON-Line System (NLS) sparingly marks selected descending nodes of unimportant trees, thus enabling natural recognition of huge groups of predictable unimportant pixels and their avoidance throughout coding. Storing of image data in one-dimensional recursive zig-zag array determines the efficiency of simple algorithms. NLS performs similarly with SPIHT. Introduction of the original SPIHT features many disadvantages; thus, many enhancements were conducted to improve this method. As it is entirely a rooted coder-decoder (codec), SPIHT method may pose benefits, namely, good image quality, high PSNR, optimization for advanced transmitted image combined efficiently to provide protection from errors, sorting of information when demanded, and decreased need for strong error correction from beginning to end.

Another advantage of SPIHT is that it downloads only small parts of the file with many practical results, thus producing compact output bit stream with large bit variation and with no requirement for an extra entropy coding. SPIHT can advance transmission of images. However, the introduction of single-bit errors may cause significantly distorted images depending on the location. The leak of organization property in a bit can lead to misinterpretation from the decoder side. This situation indirectly traces the position of important figures and poses difficulties in performing operations on dependent compressed data, significantly altering values.

[20] suggested a novel bit-rate-reduced approach to reducing the memory needed in storing remote diagnoses and quickly transmit them. This work adopted an $8 \times 8$ DCT approach to implement sub-band decomposition. The altered SPIHT was then utilized in organizing data and entropy coding. To attain DCT spectrum data in a single frequency domain, a straightforward transformation decomposes initial signals into many frequency domains, which can be compressed further by the wavelet-based algorithm. In this scheme, corresponding insignificant DCT coefficients to specific spatial locations in high-frequency sub-bands were utilized to reduce redundancy with the application of a suggested combined function associated with modified SPIHT.

Simulation results revealed that embedded DCT-SPIHT image compression reduced computational complexity to only a quarter of wavelet-based sub-band decomposition and improved reconstructed medical image quality given by PSNR; at similar bit rates, results were constant over JPEG2000 with original SPIHT. As implementation of $8 \times 8$ fast DCT hardware is commercially available, the suggested DCT-SPIHT can be executed in high-speed image coding and transmission. 
As a very popular image compression scheme, JPEG compression serves as an international standard because of its low complexity. Design and development of JPEG in 1992 intended to compress color or natural grayscale real-world images. The baseline sequential DCT-based operation mode within the JPEG standard and the sole mode is widely implemented in many image processing programs. Given the wide use of digital imaging equipment, updated JPEG2000 standard was formulated based on the strong requirement for high-performance image compression methods, which can provide complex functionality compared with JPEG standard. Based on discrete wavelet transformation (DWT) with arithmetic entropy coding, many original features are provided; these features include extracting of image parts for editing without decoding and focusing on regions of interest with sharp visual quality and definite bitrate. JPEG2000 compression standard is founded on DWT with arithmetic entropy coding.

In addition, Independent Component Analysis (ICA) has been extensively used in different applications such as blind source separation, telecommunication, medical signal processing, optimization of decision trees, analysis of financial time series, data mining Traditional ICA algorithms use unsupervised learning algorithm that models observed data set as a linear mixture of several mutually exclusive independent, non-Gaussian densities. This modeling approach assumes that there is no advance knowledge of the number of sources involved in the mixing data. This assumption makes it difficult to differentiate between those sources that contribute in the formulation of the mixing image and those that generate noises. Therefore, conventionally, ICA has been performed in the noise-less limit with noise often being dealt with as an extra source.

By applying ICA, authros in [21] has been found that images of natural scenes are well modelled when the columns of A, which can be seen as a (possibly overcomplete) basis are wavelet-like filters, and the independent sources (elements of S) have heavytailed distributions. This means that, with high probability, only a small fraction of the components of $S$ have significant values; this sparse nature of $S$ underlies the potential usefulness of overcomplete ICA to compression and denoising of natural images, in the problem of finding efficient image codes for natural scenes is addressed. The overcomplete representation gives a better approximation to the underlying statistical distribution of the data, when compared to the complete representation.

In [22], the authors addresses the use of independent component analysis (ICA) for image compression. Their goal is to study the adequacy (for lossy transform compression) of bases learned from data using ICA. They conclude that ICA provides an excellent tool for learning a coder for a specific image class, which can even be done using a single image from that class. This is an alternative to hand-tailoring a coder for a given class (as was done, for example, in the WSQ for fingerprint images). Another conclusion is that a coder learned from natural images acts like a universal coder, that is, generalizes very well for a wide range of image classes.

An ICAMM algorithm to make decomposition the basis for low-bit high-speed image compression on image sub-blocks was intrduced by [23]. The over complete independent component effect approximates the wavelet basis functions. This is different from the discrete cosine function and wavelet function in that the independent component is relevant to the data itself. When using the over complete independent component analysis to decompose the image sub-blocks, it can divide self-adaptive learning into several subgroups based on the different characteristics of the text images. Each subgroup has a set of corresponding over complete independent components. In this way, it can be more efficient to reduce the bit rate use. At the same time the information in the subgroup can also be used in image transmission based on the different encodings. The experimental results show that our proposed approach achieves low bit-rate image compression. 


\section{WIRELESS TELEMEDICINE SYSTEM}

Various functional research prototypes were developed in the last decade. These projects and prototypes vary according to their utilization. Some examples of important projects and prototypes as following:

An analyzed mobile telemedicine system challenges and provided a number of design guideline systems for designers. In utilizing commercially available $3 \mathrm{G}$ wireless data services, demonstration of two mobile telemedicine systems follow these suggested design guidelines. These systems can concurrently broadcast multiple medical information types utilizing $3 \mathrm{G}$ wireless networks. In overcoming the difficulty of restricted and unstable wireless link bandwidth, each system contains software structural design that differentiates, prioritizes, and transforms medical data, such that critical data are reliably and efficiently delivered with high quality [24].

A wireless telemedicine system design and implementation were proposed by. In this design, all physiological vital signs are transmitted to a remote medical server via cellular networks, in emergency cases, and Internet, in normal cases, for continuous monitoring. Cost of using GSM/GPRS network is reduced as only irregular cases are transmitted through cellular networks. The proposed system presents a responsive web-based interface for medical staff when observing vital signs for remote treatment.

In comparison with other systems mentioned in the introduction [25], the suggested system combines sensor unit, processing unit, and communication unit in one chip, called mobile-care unit, which is bound to a patient's body, to allow the patient to perform his/her daily activities throughout monitoring duration. The planned system provides the capability to continuously monitor patient's vital health conditions instead of separate measurements.

\section{CONCLUTION}

This paper discusses medical data compression and transmission approaches in WLAN-based telemedicine systems; these approaches were widely studied in the past years. However, existing healthcare and hospital network schemes present many challenges, which were addressed by the researchers to improve the performance of medical data transceivers through WLAN approaches. Data compression approaches should be further investigated as vital tools for decreasing communication bandwidth and saving transmission power to accept research targets.

\section{ACKNOWLEDGMENTS}

This research was funded by the Ministry of Higher Education Libya and Elmergib University.

\section{REFERENCES}

[1] Fong, B., A.C.M. Fong, and C.K. Li, Telemedicine technologies: Information technologies in medicine and telehealth. 2011: John Wiley \& Sons.

[2] Algaet, M.A., et al., Telemedicine and its application in telemedicine management. 2014, Outskirts Press, Inc.

[3] Algaet, M.A., et al. Provisioning quality of service of wireless telemedicine for e-health services. in Information \& Communication Technologies (ICT), 2013 IEEE Conference on. 2013. IEEE.

[4] Algaet, M.A., et al., Provisioning quality of service of wireless telemedicine for e-health services: A review. Wireless Personal Communications, 2014. 78(1): p. 375-406.

[5] Ahamed, S.V., Intelligent networks: recent approaches and applications in medical systems. 2013: Elsevier.

[6] Hamdy, N., Applied Signal Processing: Concepts, Circuits, and Systems. 2008: CRC Press.

[7] Perahia, E. and R. Stacey, Next generation wireless LANs: $802.11 \mathrm{n}$ and 802.11 ac. 2013: Cambridge university press. 
[8] Banerji, S. and R.S. Chowdhury, On IEEE 802.11: Wireless LAN Technology. arXiv preprint arXiv:1307.2661, 2013.

[9] Jiang, D. and L. Delgrossi. IEEE 802.11 p: Towards an international standard for wireless access in vehicular environments. in Vehicular Technology Conference, 2008. VTC Spring 2008. IEEE. 2008. IEEE.

[10] Luo, F.-L., Digital Front-End in Wireless Communications and Broadcasting: circuits and signal processing. 2011: Cambridge University Press.

[11] Nijsure, Y., et al. Location-aware spectrum and power allocation in joint cognitive communicationradar networks. in Cognitive Radio Oriented Wireless Networks and Communications (CROWNCOM), 2011 Sixth International ICST Conference on. 2011. IEEE.

[12] Chang, F., K. Onohara, and T. Mizuochi, Forward error correction for $100 \mathrm{G}$ transport networks. IEEE Communications Magazine, 2010. 48(3).

[13] Candy, P., Healthcare knowledge management: issues, advances and successes. 2010: Springer Science \& Business Media.

[14] Algaet, M.A., et al., Development of Robust Medical Image Transmission via Wi-Fi IEEE 802.11 b in the Hospital Area. Wireless Personal Communications, 2017. 95(2): p. 1617-1634.

[15] Sen, S., et al. Avoiding multipath to revive inbuilding WiFi localization. in Proceeding of the 11th annual international conference on Mobile systems, applications, and services. 2013. ACM.

[16] Jayapandian, C.P., et al. Cloudwave: distributed processing of "Big Data" from electrophysiological recordings for epilepsy clinical research using Hadoop. in AMIA Annual Symposium Proceedings. 2013. American Medical Informatics Association.

[17] Paul, R.P., C.K. Modi, and R.K. Kher, A simple and novel algorithm for medical image compression. International Journal of Biomedical Engineering and Technology, 2012. 10(4): p. 333-350.

[18] Gersho, A. and R.M. Gray, Vector quantization and signal compression. Vol. 159. 2012: Springer Science \& Business Media.

[19] Khan, E. and M. Ghanbari. Error detection and correction of transmission errors in SPIHT coded images. in Image Processing. 2002. Proceedings. 2002 International Conference on. 2002. IEEE.

[20] Kumar, G., et al., A Review: DWT-DCT Technique and Arithmetic-Huffman Coding based Image Compression. IJ Engineering and Manufacturing, 2015. 3: p. 20-33.

[21] Puga, A. and A. Alves. An experiment on comparing PCA and ICA in classical transform image coding. in ICA. 1998.

[22] Ferreira, A.J. and M.A. Figueiredo, On the use of independent component analysis for image compression. Signal Processing: Image Communication, 2006. 21(5): p. 378-389.

[23] Peng, C.-C., C.-H. Kao, and S.-P. Hsieh. Fast image compression using over-complete ICAMM. in Control Automation and Systems (ICCAS), 2010 International Conference on. 2010. IEEE.

[24] Mustapha, A., et al., Towards nonuniform illumination face enhancement via adaptive contrast stretching. Multimedia Tools and Applications, 2017. 76(21): p. 21961-21999.

[25] Hadzievski, L., et al., A novel mobile transtelephonic system with synthesized 12-lead ECG. IEEE transactions on information technology in biomedicine, 2004. 8(4): p. 428-438. 
International Journal of Grid and Distributed Computing Vol. 12, No. 2 (2019) 